\title{
Research on Extraction Method of LOS rate of Rotating Bomb Full strap-down seeker Based on Kalman Filter
}

\author{
Fang Hongsong ${ }^{a}$, Liu Yongshan ${ }^{b}$ \\ Beijing Institute of Technology,Beijing 100081,China; \\ a1074019708@qq.com, bliuysh@bit.edu.cn.
}

Keywords: Rotating missile, Full strap-down seeker, Inertial line of sight rate extraction, Kalman filter.

\begin{abstract}
In this paper, the rotating bomb full strap-down seeker is taken as the research object. We focus on the extraction method of LOS rate of full strap-down seeker. The structure of the full strap-down seeker is completely different from that of the conventional platform seeker. Its measurement system is fixed together with the missile body. So the seeker can only get the measurement information under the missile coordinate system. And the measurement information is coupled with the missile posture information, can't be directly used for the control of the missile. According to the conversion relationship between the coordinate system, the mathematical model and decoupling model of LOS are established. In this way, the mathematical model of the LOS angle rate extraction is further established. At the same time, two kinds of Kalman filter methods, EKF and UKF, are used to design the Kalman filter to filter the extracted LOS containing noise information. The validity of the LOS rate extraction mathematical model is verified on MATLAB. And the mathematical simulation on MATLAB verify that the designed Kalman filter can effectively extract the line of sight rate information.
\end{abstract}

\section{Introduction}

The Rotating missile, as a member of the missile family, has advantages of the system structure is simple, low cost, high reliability, etc. And its own spin movement will bring some convenience, can overcome some problems, such as thrust eccentricity, the control point spread, uneven distribution of missile quality, random gust ${ }^{[1]}$. Compared with the traditional Tilted and stable missiles, rotating bomb is more suitable for the development trend of missile miniaturization and precision, has broad prospects for development.

In this study, the rotary missile guide is a full strap-down laser seeker, which has many advantages such as small volume, light weight, low complexity, high reliability, large instantaneous field of view, unrestricted LOS rate and low cost. The measuring system of laser seeker is linked together with missile body, and the measurement information is the LOS angle under the missile body coordinate system, cannot be directly used for rotating bomb guidance control ${ }^{[2]}$. It is necessary to solve the LOS angle information of the seeker under the inertial system, and to extract the useful LOS rate information by the filter method.

\section{Establishment of Inertial Line of Sight Extraction Model}

The full strap-down In this paper, the following coordinate systems are used in the study of the extraction method of the LOS angle of the full strap-down seeker: Ground coordinate system (Inertial coordinate system) $A x y z$, Projectile coordinate system $O x_{1} y_{1} z_{1}$, Line-of-sight coordinate system $O x_{s} y_{s} z_{s}$, Body line of sight coordinate system $O x_{T} y_{T} z_{T}$. The angles which used in this paper 
are pitch angle $\vartheta$, yaw angle $\psi$, roll angle $\gamma$, line of sight azimuth $q_{\lambda}$, LOS elevation angle $q_{\gamma}$, body LOS azimuth $q_{\beta}$, body LOS elevation angle $q_{\alpha}$. The definition of the relative coordinate system and angle are described in the literature [3].

laser seeker can't directly get the LOS angle under the inertial system information as the traditional platform seeker, due to itself is associated with the rotating bomb body. But rotating bomb guidance control need is inertial line-of-sight information, so we need to calculate the extraction mathematical model of the inertial line-of-sight angle of the laser seeker. At the same time, the body LOS angle information measured by the seeker measurement system is coupled with the inertial line-of-sight information and the projectile attitude information ${ }^{[4-5]}$. Therefore, the observation equation based on the line-of-sight angle information ( $q_{\alpha}$ and $q_{\beta}$ ) containing noise was established. Based on the inertial line-of-sight angle $\left(q_{\gamma}, q_{\lambda}\right)$ and the inertial line-of-sight rate $\left(\dot{q}_{\gamma}\right.$, $\dot{q}_{\lambda}$ ), the state equation was established. Thus the establishment of inertial line of sight extraction model was completed.

\subsection{The State Equation}

The distance between the missile and the target is defined as $r$, so the approaching speed of the missile can be expressed as

$$
\mathbf{V}=\dot{\mathbf{r}}=\dot{r} i_{s}+r \dot{i}_{s}
$$

In the formula (1), respectively, $i_{s}, j_{s}, k_{s}$ are the unit vector of the line of sight coordinate $O x_{s}$ axis, $O y_{s}$ axis and $O z_{s}$ axis. The rotational angular velocity of the line of sight coordinate system relative to the geographic coordinate system is

$$
\omega_{s}=\dot{q}_{\lambda} \sin q_{\gamma} i_{s}+\dot{q}_{\lambda} \cos q_{\gamma} j_{s}+\dot{q}_{\gamma} k_{s}
$$

In the line of sight coordinate system, there is

$$
\begin{aligned}
\dot{i}_{s} & =\omega_{s} \times \dot{i}_{s} \\
& =\left|\begin{array}{ccc}
i_{s} & j_{s} & k_{s} \\
\dot{q}_{\lambda} \sin q_{\gamma} & \dot{q}_{\lambda} \cos q_{\gamma} & \dot{q}_{\gamma} \\
1 & 0 & 0
\end{array}\right| \\
& =\dot{q}_{\gamma} j_{s}-\dot{q}_{\lambda} \cos q_{\gamma} k_{s}
\end{aligned}
$$

And similarly, we can get the following two formulas.

$$
\begin{gathered}
\dot{j}_{s}=\dot{q}_{\lambda} \sin q_{\gamma} k_{s}-\dot{q}_{\gamma} i_{s} \\
\dot{k}_{s}=\dot{q}_{\lambda} \cos q_{\gamma} i_{s}-\dot{q}_{\lambda} \sin q_{\gamma} j_{s}
\end{gathered}
$$

By differentiating the relative velocity of the missile and target, the relative acceleration of the projectile is obtained.

$$
\mathbf{a}=\dot{\mathbf{V}}=\left[\begin{array}{c}
\ddot{r}-r \dot{q}_{\gamma}{ }^{2}-r \dot{q}_{\lambda}{ }^{2} \cos ^{2} q_{\gamma} \\
2 \dot{r} \dot{q}_{\gamma}+r \ddot{q}_{\gamma}+r \dot{q}_{\lambda}{ }^{2} \sin q_{\gamma} \cos q_{\gamma} \\
2 r \dot{q}_{\gamma} \dot{q}_{\lambda} \sin q_{\gamma}-2 \dot{r} \dot{q}_{\lambda} \cos q_{\gamma}-r \ddot{q}_{\lambda} \cos q_{\gamma}
\end{array}\right]
$$

If the target is a fixed target on the ground, the relative acceleration between the missile and target is zero. So we can deduce the following formula.

$$
\left\{\begin{array}{c}
\ddot{r}=r \dot{q}_{\gamma}^{2}+r \dot{q}_{\lambda}^{2} \cos ^{2} q_{\gamma} \\
\ddot{q}_{\gamma}=-\frac{2 \dot{r}}{r} \dot{q}_{\gamma}-\dot{q}_{\lambda}^{2} \sin q_{\gamma} \cos q_{\gamma} \\
\ddot{q}_{\lambda}=-\frac{2 \dot{r}}{r} \dot{q}_{\lambda}+2 \dot{q}_{\gamma} \dot{q}_{\lambda} \tan q_{\gamma}
\end{array}\right.
$$


The state of the state equation is $X=\left[\begin{array}{llll}q_{\gamma} & \dot{q}_{\gamma} & q_{\lambda} & \dot{q}_{\lambda}\end{array}\right]^{T}$, so the state equation is

$$
\left\{\begin{array}{c}
\dot{X}_{1}=X_{2} \\
\dot{X}_{2}=-\frac{2 V}{r} X_{2}-X_{4}^{2} \sin X_{1} \cos X_{1} \\
\dot{X}_{3}=X_{4} \\
\dot{X}_{4}=2 X_{2} X_{4} \tan X_{1}-\frac{2 V}{r} X_{4}
\end{array}\right.
$$

\subsection{The Observation Equation}

The coordinates of the target in the body line of sight coordinate system and the line of sight coordinate system are $\left[\begin{array}{lll}r & 0 & 0\end{array}\right]^{T}$. According to the conversion relationship between the coordinate system, the coordinates of the target in the body coordinate system and the ground coordinate system can be obtained respectively.

$$
\begin{gathered}
{\left[\begin{array}{l}
x_{1} \\
y_{1} \\
z_{1}
\end{array}\right]=L\left(q_{\beta}, q_{\alpha}\right)\left[\begin{array}{l}
r \\
0 \\
0
\end{array}\right]=\left[\begin{array}{l}
r \cos q_{\alpha} \cos q_{\beta} \\
r \sin q_{\alpha} \\
-r \cos q_{\alpha} \sin q_{\beta}
\end{array}\right]} \\
{\left[\begin{array}{l}
x \\
y \\
z
\end{array}\right]=L\left(q_{\lambda}, q_{\gamma}\right)\left[\begin{array}{l}
r \\
0 \\
0
\end{array}\right]=\left[\begin{array}{l}
r \cos q_{\gamma} \cos q_{\lambda} \\
r \sin q_{\gamma} \\
-r \cos q_{\gamma} \sin q_{\lambda}
\end{array}\right]}
\end{gathered}
$$

In order to facilitate the calculation, we set a coefficient matrix.

$$
L(\gamma, \vartheta, \psi)=\left[\begin{array}{lll}
R_{11} & R_{12} & R_{13} \\
R_{21} & R_{22} & R_{23} \\
R_{31} & R_{32} & R_{33}
\end{array}\right]
$$

According to the conversion between the ground coordinate system and the body coordinate system, the formula (12) can be obtained.

$$
\left[\begin{array}{l}
\cos q_{\alpha} \cos q_{\beta} \\
\sin q_{\alpha} \\
-\cos q_{\alpha} \sin q_{\beta}
\end{array}\right]=\left[\begin{array}{l}
R_{11} \cos q_{\gamma} \cos q_{\lambda}+R_{12} \sin q_{\gamma}-R_{13} \cos q_{\gamma} \sin q_{\lambda} \\
R_{21} \cos q_{\gamma} \cos q_{\lambda}+R_{22} \sin q_{\gamma}-R_{23} \cos q_{\gamma} \sin q_{\lambda} \\
R_{31} \cos q_{\gamma} \cos q_{\lambda}+R_{32} \sin q_{\gamma}-R_{33} \cos q_{\gamma} \sin q_{\lambda}
\end{array}\right]
$$

To solve the equation (12), the observation equation can be obtained.

$$
\begin{gathered}
q_{\alpha}=\arcsin \left(R_{21} \cos q_{\gamma} \cos q_{\lambda}+R_{22} \sin q_{\gamma}-R_{23} \cos q_{\gamma} \sin q_{\lambda}\right) \\
q_{\beta}=\arctan \left(\frac{-R_{31} \cos q_{\lambda}-R_{32} \tan q_{\gamma}+R_{33} \sin q_{\lambda}}{R_{11} \cos q_{\lambda}+R_{12} \tan q_{\gamma}-R_{13} \sin q_{\lambda}}\right)
\end{gathered}
$$

\section{Experimental Simulation Verification}

The LOS angle information measured by the full strap-down laser seeker contains external noise and interference information. If the inertial line of sight is used for the guidance of the missile directly, will cause the guidance precision of missile is very poor, and even could make the missile miss distance. In order to make the missile can hit the target accurately, we need to select the appropriate filtering method, filter out the noise and interference in the line of sight information, and extract useful line of sight rate information ${ }^{[6-8]}$.

From the state equation and the observation equation in the second section, it can be seen that the signal of the inertial line of sight extraction model is a random signal. For random signal, the conventional filtering method can't achieve a good filtering effect, so this paper adopts Kalman filter to the line of sight Angle signal filtering and verify with program in MATLAB. The simulation 
of this mathematical experiment is for the situation that the target position has a binding error and navigation system has measurement error. In the ground coordinate system, the actual target position is ) 0,2.5,0 and the target position coordinate measurement error is) 100, 50,60 . The target coordinate values are bound to the missile navigation system. The simulation results of EKF and UKF are shown in fig. 1-4 respectively.

From the following results, the filter value of EKF and UKF is close to the true value, can be applied to guidance control of rotating bomb. It is proved that the proposed line of sight angular rate extraction algorithm based on Kalman filter is effective. And from the RMS error of the error between the filter value and the true value, it can be seen that the filtering effect of UKF is better than EKF.

The RMS error of the error between the filter value and the true value is shown in table 1.

Table 1 The RMS error of the error between the filter value and the true value

\begin{tabular}{|c|c|c|}
\hline & EKF & UKF \\
\hline LOS altitude angle rate & 0.3422 & 0.3368 \\
\hline LOS azimuth rate & 0.1779 & 0.1675 \\
\hline
\end{tabular}

Simulation results of EKF:

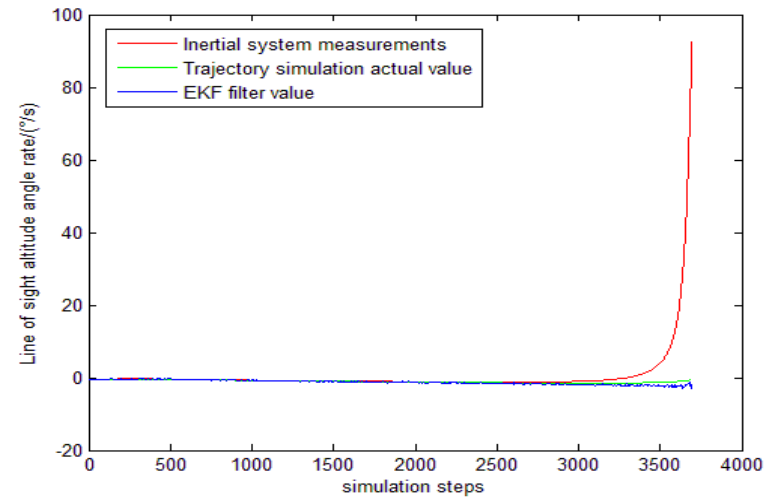

Figure 1.EKF line of sight altitude angle rate filter results

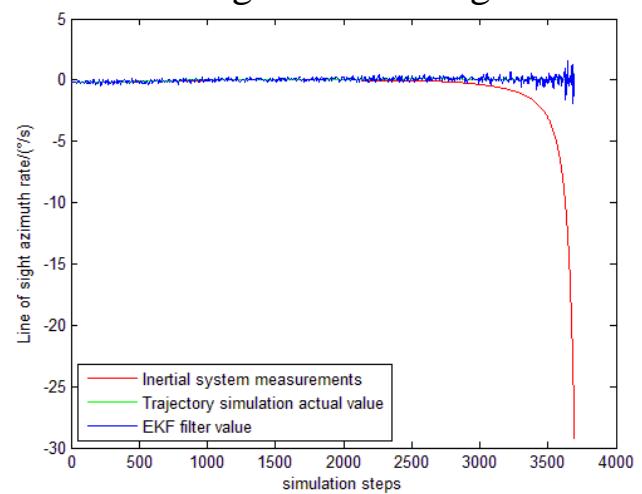

Figure 2.EKF line of sight azimuth rate filter results

Simulation results of UKF:

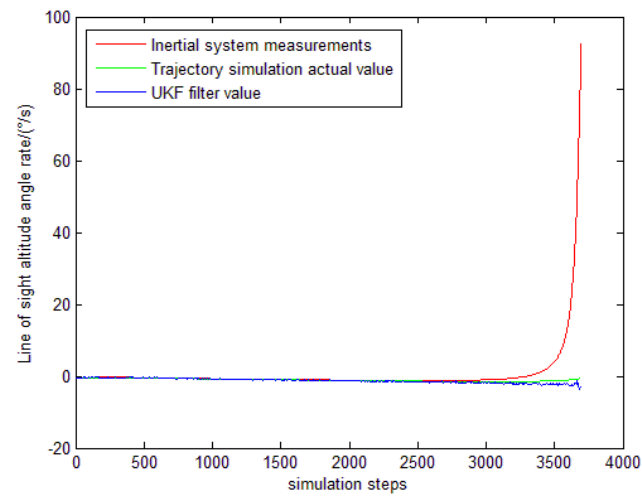

Figure 3.UKF line of sight altitude angle rate filter results 


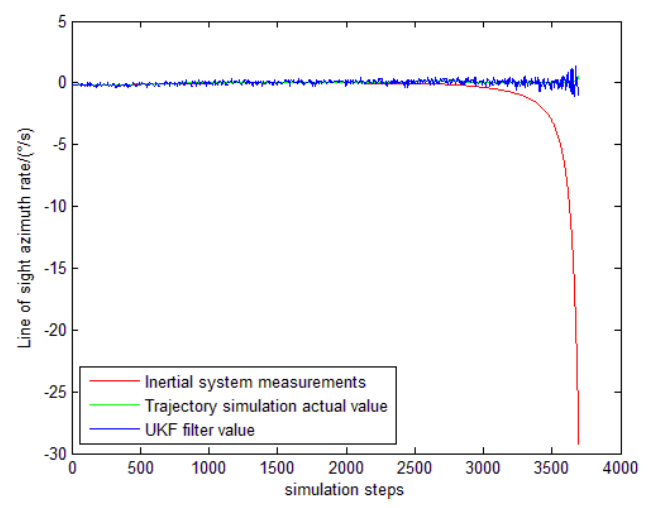

Figure 4.UKF line of sight azimuth rate filter results

\section{Conclusion}

This paper introduces the extraction method of LOS rate of rotating bomb full strap-down seeker based on Kalman filter. The method can solve coupling problem of the line of sight information and missile posture information, and solve the coupling problem of the pitch angle and yaw angle, and extract the useful inertial LOS rate information. The results of MATLAB simulation show that the inertial LOS rate information can be used to replace the actual inertial line of sight rate information in the guidance process of the rotating missile. This has some reference significance for the study of rotating bomb.

\section{References}

[1]Tingting Sun. Research on Key Technology of Full Strapdown Guidance System[D]. Changchun: Graduate School of Chinese Academy of Sciences (Changchun Institute of Optics and Fine Mechanics and Physics),2016.

[2]Pengpeng Xiu,Xiaobing Li,Yuzhe Yang. A Method of Calculating the Line of Sight[J]. Photoelectric engineering, 2012,39 (7): 75-80.

[3]Qingfang Qian,Ruixiong Lin,Yanan Zhao. Missile flight mechanics[M]. Beijing: Beijing Polytechnic University Press, 2013.

[4] WALDMANN J.Line-of-sight rate estimation and linearizing control of an imaging seeker in a tactical missile guided by proportional navigation [J].IEEE Transactions on control systems technology,2002,10(4):56-67.

[5]Jingjing Li. Research of LOS Rate Estimation Method For Strap-down Imaging Seeker[D]. Harbin: Harbin Institute of Technology .2008.

[6] SUN T,CHU H,ZHANG B,et al.Line-of-Sight Rate Estimation Based on UKF for Strapdown Seeker [J].Mathematical Problems in Engineering,2015, 2015 (1) :1-14.

[7]Yongyuan Qin,Hongyue Zhang,Shuhua Wang. Kalman Filtering and Combined Navigation Principle[M]. Xi'an: Northwestern Polytechnical University Press. 2012.

[8]WANG P,ZHANG K.Research on Line-of-Sight rate extraction of strapdown seeker;proceedings of the Control Conference,2014 33rd Chinese,F,2014[C].IEEE. 